\title{
Mycobacterium tuberculosis lineage 4 comprises globally distributed and geographically restricted sublineages
}

\author{
David Stucki ${ }^{1,2,57}$, Daniela Brites ${ }^{1,2,57}$, Leïla Jeljeli ${ }^{3,4,57}$, Mireia Coscolla ${ }^{1,2}$, Qingyun Liu ${ }^{5}$, Andrej Trauner ${ }^{1,2}$,
} Lukas Fenner $^{1,2,6}$, Liliana Rutaihwa ${ }^{1,2}$, Sonia Borrell ${ }^{1,2}$, Tao Luo ${ }^{7}$, Qian Gao ${ }^{5}$, Midori Kato-Maeda ${ }^{8}$, Marie Ballif ${ }^{1,2,6}$, Matthias Egger ${ }^{6}$, Rita Macedo ${ }^{9}$, Helmi Mardassi ${ }^{4}$, Milagros Moreno ${ }^{10}$, Griselda Tudo Vilanova ${ }^{11}$, Janet Fyfe ${ }^{12}$, Maria Globan ${ }^{12}$, Jackson Thomas ${ }^{13}$, Frances Jamieson ${ }^{14}$, Jennifer L Guthrie ${ }^{14}$, Adwoa Asante-Poku ${ }^{15}$, Dorothy Yeboah-Manu $^{15}$, Eddie Wampande ${ }^{16}$, Willy Ssengooba ${ }^{16,17}$, Moses Joloba ${ }^{16}$, W Henry Boom ${ }^{18}$, Indira Basu ${ }^{19}$, James Bower ${ }^{19}$, Margarida Saraiva ${ }^{20,21}$, Sidra E G Vasconcellos ${ }^{22}$, Philip Suffys ${ }^{22}$, Anastasia Koch ${ }^{23}$, Robert Wilkinson $^{23-25}$, Linda Gail-Bekker ${ }^{23}$, Bijaya Malla ${ }^{1,2}$, Serej D Ley ${ }^{1,2,26}$, Hans-Peter Beck ${ }^{1,2}$, Bouke C de Jong ${ }^{27}$, Kadri Toit ${ }^{28}$, Elisabeth Sanchez-Padilla ${ }^{29}$, Maryline Bonnet ${ }^{29}$, Ana Gil-Brusola ${ }^{30}$, Matthias Frank ${ }^{31}$,

Veronique N Penlap Beng ${ }^{32}$, Kathleen Eisenach ${ }^{33}$, Issam Alani ${ }^{34}$, Perpetual Wangui Ndung' ${ }^{35}$, Gunturu Revathi ${ }^{36}$, Florian Gehre ${ }^{27,37}$, Suriya Akter ${ }^{27}$, Francine Ntoumi ${ }^{31,38}$, Lynsey Stewart-Isherwood ${ }^{39}$, Nyanda E Ntinginya ${ }^{40}$, Andrea Rachow ${ }^{41}$, Michael Hoelscher ${ }^{41}$, Daniela Maria Cirillo ${ }^{42}$, Girts Skenders ${ }^{43}$, Sven Hoffner ${ }^{44}$, Daiva Bakonyte ${ }^{45}$, Petras Stakenas ${ }^{45}$, Roland Diel ${ }^{46}$, Valeriu Crudu ${ }^{47}$, Olga Moldovan ${ }^{48}$, Sahal Al-Hajoj ${ }^{49}$, Larissa Otero $^{50}$, Francesca Barletta ${ }^{50}$, E Jane Carter ${ }^{51,52}$, Lameck Diero ${ }^{52}$, Philip Supply ${ }^{53}$, Iñaki Comas ${ }^{54,55}$, Stefan Niemann ${ }^{3,56} \&$ Sebastien Gagneux ${ }^{1,2}$

Generalist and specialist species differ in the breadth of their ecological niches. Little is known about the niche width of obligate human pathogens. Here we analyzed a global collection of Mycobacterium tuberculosis lineage 4 clinical isolates, the most geographically widespread cause of human tuberculosis. We show that lineage 4 comprises globally distributed and geographically restricted sublineages, suggesting a distinction between generalists and specialists. Population genomic analyses showed that, whereas the majority of human T cell epitopes were conserved in all sublineages, the proportion of variable epitopes was higher in generalists. Our data further support a European origin for the most common generalist sublineage. Hence, the global success of lineage 4 reflects distinct strategies adopted by different sublineages and the influence of human migration.

Ecologists distinguish between generalists and specialists on the basis of the width of an organism's ecological niche ${ }^{1}$. In infectious diseases, the niche of a given pathogen is determined by host range and the agent's capacity to survive in the environment ${ }^{2}$. Some microbes are obligate pathogens restricted to one or several host species ${ }^{3,4}$; others are mainly free living and are only occasionally pathogenic ${ }^{5}$. Little is known about the niche width of obligate human pathogens ${ }^{3}$. The causative agent of tuberculosis, known as the Mycobacterium tuberculosis complex (MTBC), is an obligate pathogen that comprises seven phylogenetic lineages adapted to humans and two lineages adapted to various wild and domestic animal species ${ }^{6}$. Some human-adapted MTBC lineages have received particular attention. For example, lineage 2, which includes the Beijing family of strains, has repeatedly been associated with drug resistance ${ }^{7}$. Lineage 2 likely originated in East Asia ${ }^{8,9}$ and has recently been expanding in some parts of the world $^{10}$. By contrast, lineages 5 and 6 (also known as Mycobacterium africanum West Africa I and II) and lineage 7 are largely restricted to West and East Africa, respectively ${ }^{11,12}$. The observation that the human-adapted MTBC population is phylogeographically structured has led to the hypothesis that the different lineages might be adapted to particular human populations ${ }^{13}$. Support for this notion comes from the observation that sympatric host-pathogen associations in human tuberculosis remain stable over time, even in metropolitan settings where host and pathogen populations intermix ${ }^{14-17}$. Moreover, sympatric host-pathogen associations are perturbed in patients coinfected with $\mathrm{HIV}^{14}$, indicating that, in the context of reduced host immune competence, the different lineages can successfully infect and cause disease irrespective of the host genetic background.

Contrary to the other main human-adapted MTBC lineages, lineage 4 exists at high frequencies on all inhabited continents ${ }^{18}$. It is hence geographically the most widespread cause of human tuberculosis ${ }^{19}$. Yet, the reasons for this global success are unknown. Lineage 4 has been shown to exhibit enhanced virulence in macrophage and animal models of infection, albeit with much variation among

A full list of affiliations appears at the end of the paper.

Received 3 August 2016; accepted 27 September 2016; published online 31 October 2016; doi:10.1038/ng.3704 
different lineage 4 strains ${ }^{19,20}$. Moreover, molecular epidemiological studies have reported considerable variation in the transmission success of different lineage 4 strains in clinical settings ${ }^{19}$. These observations suggest that lineage 4 is genetically and phenotypically diverse, and this diversity might determine the epidemiology of different lineage 4 subtypes in different parts of the world. The purpose of this study is to gain a better understanding of the global population structure of lineage 4 and the evolutionary forces that have contributed to the success of lineage 4 across the world. For this purpose, we combined large-scale SNP typing with targeted whole-genome sequencing of a global collection of lineage 4 clinical isolates.

\section{RESULTS}

\section{MTBC lineage 4 comprises ten separate sublineages}

We first analyzed 72 published genome sequences of lineage 4 clinical strains from global sources ${ }^{21,22}$. These strains harbored 9,455 variable single-nucleotide positions that divided lineage 4 into ten sublineages (L4.1.1 to L4.10 in Fig. 1a and Supplementary Fig. 1). We used four complementary approaches to validate these sublineages. First, we performed principal-component analysis, which showed clear separation of seven sublineages (L4.1.1, L4.1.3, L4.1.2, L4.2, L4.3, L4.4 and L4.10; Supplementary Fig. 2). Sublineages L4.5, L4.6.1/Uganda and L4.6.2/ Cameroon were less clearly separated. Second, we found that the mean pairwise genetic distance between pairs of isolates within the sublineages was significantly lower than that between sublineages (276 SNPs versus 602 SNPs; Wilcoxon rank-sum test, $P<0.0001$; Supplementary Fig. 3). Overall, the mean pairwise SNP distance between any two strains was 565 SNPs. Third, we calculated pairwise fixation indexes $\left(F_{\mathrm{ST}}\right)$ to evaluate the degree of population differentiation. All $F_{\mathrm{ST}}$ values between the sublineages were larger than 0.33 (Supplementary Table 1), indicating that these populations are separated. Fourth, we mapped previously reported phylogenetic markers onto our genomebased phylogenetic tree ${ }^{15,23-28}$. Most of these markers were congruent with our sublineage definition (Supplementary Fig. 1).

\section{Sublineages differ in their phylogeographical distributions}

Because the MTBC exhibits limited sequence variation and little ongoing horizontal gene exchange, SNP homoplasies are extremely rare, making SNPs ideal phylogenetic markers ${ }^{29}$. We further scrutinized the 9,455 variable positions among the 72 MTBC lineage 4 genomes and found 51 to 277 specific for one of each of the ten sublineages. All of these variable positions were mutually exclusive: that is, they showed no homoplasy. We selected a subset of these sublineage-specific SNPs and used them to screen a global collection of 3,366 lineage 4 clinical isolates from 100 countries using various genotyping platforms ${ }^{30-35}$. First, we developed a novel sublineage-specific multiplexed SNP typing assay using the Luminex platform as previously reported $^{36}$, and we used this method to screen 2,001 isolates (Supplementary Table 2). In addition, we screened 741 isolates using the Sequenom MassARRAY platform (Supplementary Table 3) ${ }^{37}$ and 624 isolates by PCR and Sanger sequencing (Supplementary Table 4). Overall, 3,181 of 3,366 (94.5\%) lineage 4 isolates were successfully assigned to a sublineage (Supplementary Table 5). An additional 92 of 3,366 (2.7\%) isolates harbored the reference allele for all sublineages, indicating that they belonged to one or several additional and unknown sublineages. For the remaining 93 of 3,366 $(2.8 \%)$ isolates, no classification could be obtained for various technical reasons. Among the 3,181 lineage 4 isolates assigned to one of the ten sublineages, L4.3/LAM was the most frequent, accounting for $20.3 \%$, followed by L4.6.1/Uganda (14.2\%), L4.10/PGG3 (11.9\%), L4.4 (10.1\%) and L4.1.2/Haarlem (9.9\%) (Fig. 1b).
Mapping the proportion of each sublineage by country showed that the sublineages differed in their geographical distributions (Fig. 2). Specifically, L4.1.2/Haarlem, L4.3/LAM and L4.10/PGG3 occurred globally (Fig. 3a and Supplementary Fig. 4). By contrast,
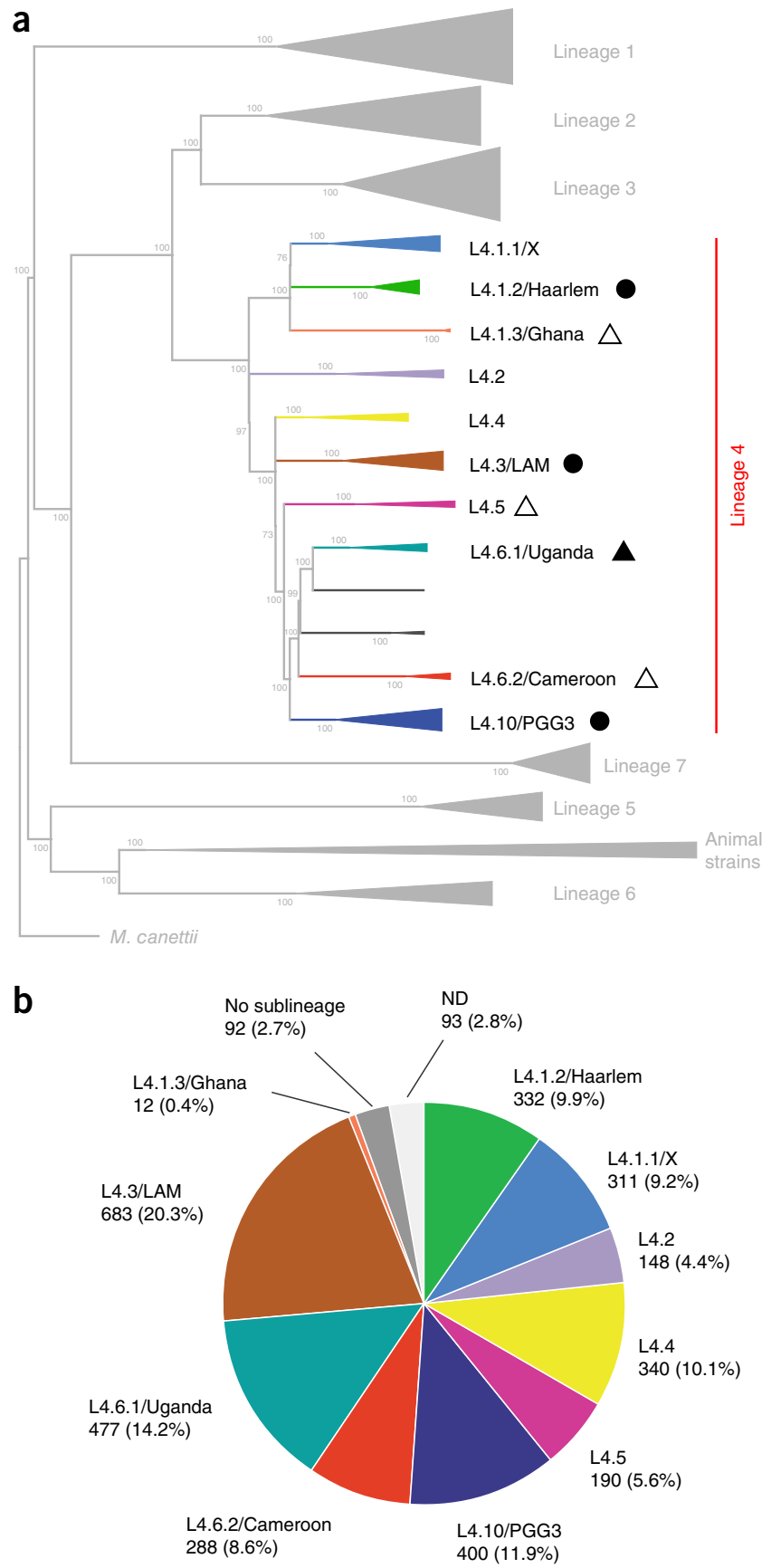

Figure 1 Definition and global frequency of lineage 4 sublineages. (a) We defined ten sublineages on the basis of the analysis of 72 MTBC lineage 4 genome sequences published previously21,22. Sublineages were labeled according to Coll et al. ${ }^{27}$ (whenever possible) and previous designations based on spoligotyping (Supplementary Fig. 1). Black triangles represent sublineages identified as specialists, and black circles represent generalists. Filled shapes correspond to sublineages for which we performed deep genomic analyses. (b) Global proportion of each sublineage. A total of 3,366 MTBC lineage 4 isolates were screened for sublineage-specific SNPs. L4.3/LAM was the most frequent sublineage globally. ND, not defined. 

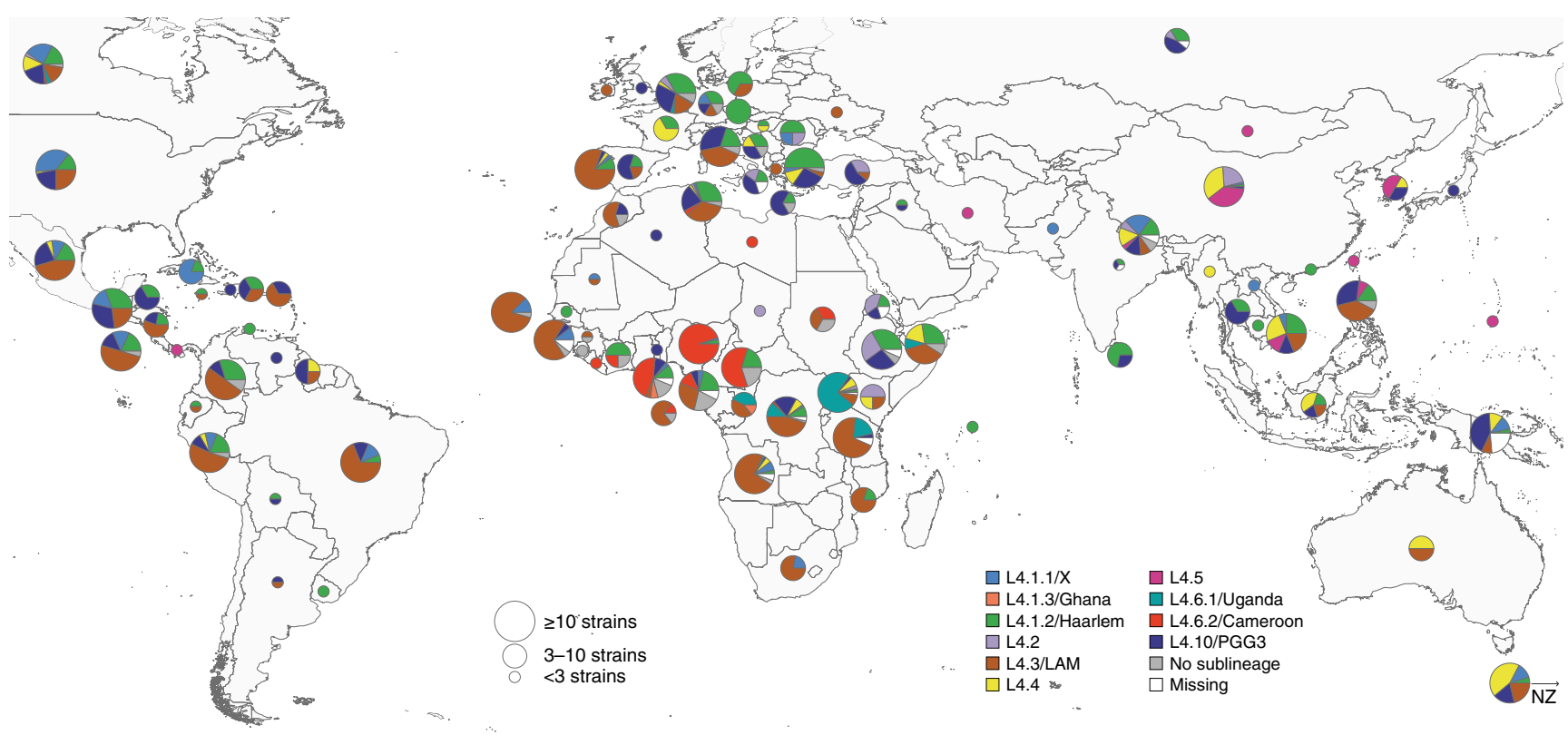

Figure 2 Global distribution of lineage 4 sublineages. Pie charts show the proportions of the ten lineage 4 sublineages among all MTBC lineage 4 isolates in each country. Circle sizes correspond to the number of isolates analyzed per country. A total of 3,366 MTBC lineage 4 isolates were included. Color codes are as in Figure 1.

L4.1.3/Ghana, L4.5, L4.6.1/Uganda and L4.6.2/Cameroon occurred at high frequencies in specific regions of Africa or Asia, and were almost completely absent from Europe and the Americas (Fig. 3b). The geographical spread of the three remaining sublineages was intermediate (Fig. 2 and Supplementary Figs. 4 and 5). L4.1.1/X mainly occurred in the Americas and in lower proportions in a few countries of southern Africa, Asia and Europe. L4.2 and L4.4 occurred in high proportions among isolates from particular countries in Asia and Africa, but were largely absent from the Americas (Fig. 2 and Supplementary Figs. 4 and 5). A similar pattern of sublineage distribution was observed when normalizing by tuberculosis prevalence ${ }^{38}$ and country surface area (Supplementary Fig. 6).

Populations that occupy a broader variety of environments may exhibit a wider geographical distribution. Humans differ in their susceptibility to tuberculosis ${ }^{39}$, and human genetic diversity may thus determine the width of the ecological niche accessible to different MTBC genotypes ${ }^{40,41}$. The geographical restriction of particular MTBC genotypes might reflect local adaptation of these pathogen variants to the corresponding human host populations ${ }^{13,15}$. Such a sympatric host-pathogen association in human tuberculosis is compatible with the 'local' sublineages observed here and supports the notion that these sublineages represent ecological specialists. By contrast, the three 'global' sublineages could represent generalists capable of infecting and causing disease in many different human populations. This notion was supported by the fact that the three generalist sublineages L4.1.2/Haarlem, L4.3/LAM and L4.10/PGG3 were observed in 49,47 and 47 countries, respectively, whereas the specialist sublineages L4.1.3/Ghana, L4.5, L4.6.1/Uganda and L4.6.2/Cameroon were only found in a few countries each $(3,7,9$ and 10 countries, respectively). The country frequencies of the remaining three sublineages, L4.1/X, L4.2/Ural and L4.4, were intermediate (27, 14 and 26 countries, respectively) (Supplementary Fig. 4).

The different geographical distributions of generalist and specialist sublineages could be due to intrinsic biological factors, extrinsic factors such as human migration, or both. Hence, we next performed various population genomic analyses to explore the genomic characteristics of these lineage 4 generalists and specialists, as well as the role of human migration in the global spread of the most successful generalist sublineage.

\section{Genomic features of generalist and specialist sublineages}

The geographical and niche distributions of populations can be correlated with their genetic variability or with that of their ancestors. One possible reason for the restricted host range of the specialist sublineages might be historical; that is, the ancestor populations of the extant specialist populations may have harbored more deleterious mutations, restricting host range. To assess this possibility, we characterized the mutations that contributed to the divergence of the different sublineages; these mutations are variants that have become fixed during the evolution of the sublineages. We focused on substitutions that occurred in all isolates of any of the generalist sublineages (L4.1.2/Haarlem, L4.3/LAM and L4.10/PGG3) and compared them to substitutions that occurred in all isolates of any of the three specialist sublineages (L4.6.1/Uganda, L4.5 and L4.6.2/Cameroon). We identified nonsynonymous SNPs predicted to have a functional effect using SIFT ${ }^{42}$. We found that, overall, the specialist and generalist sublineages showed similar proportions of fixed substitutions (among all substitutions) predicted to affect gene function 23 (23.0\% versus $20.6 \%$; $\chi^{2}$ test, $P=0.62$; Supplementary Table 6 ), suggesting that the mutational load of the ancestor populations did not differ significantly between generalists and specialists.

Small populations with restricted geographical ranges are expected to have reduced levels of genetic diversity ${ }^{43}$. Thus, one possible restriction to niche expansion by specialist sublineages could be that these sublineages have low genetic diversity, precluding adaptation to new hosts. We characterized the genetic diversity associated with the process of diversification in lineage 4 generalists and specialists, focusing on L4.3/LAM and L4.6.1/Uganda, which globally were the most frequent generalist and specialist sublineages of lineage 4 in our data set, respectively (Fig. 1b). We analyzed the whole-genome 
a
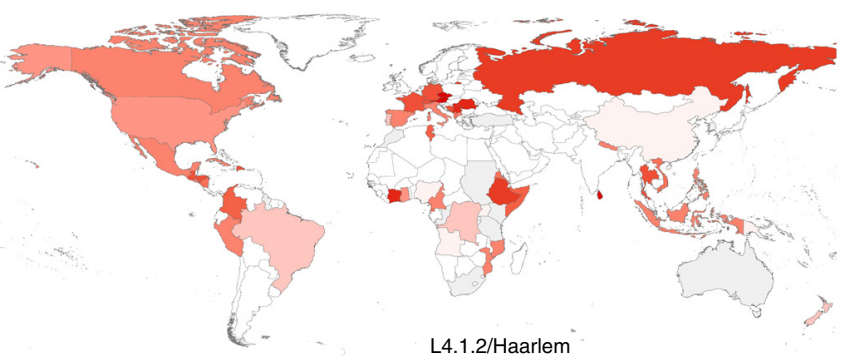

L4.1.2/Haarlem

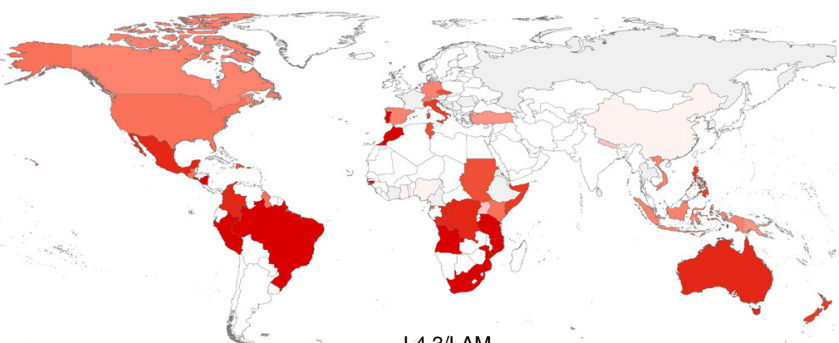

L4.3/LAM
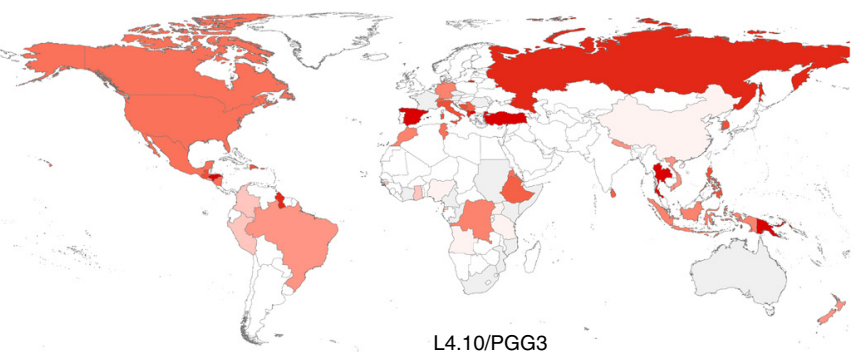

L4.10/PGG3

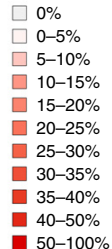

$\square 0 \%$
$\square 0-5 \%$
$\square-10 \%$
$\square 10-15 \%$
$\square 15-20 \%$
$\square 20-25 \%$
$\square 25-30 \%$
$\square 30-35 \%$
$\square 3-40 \%$
$40-50 \%$
$50-100 \%$ b
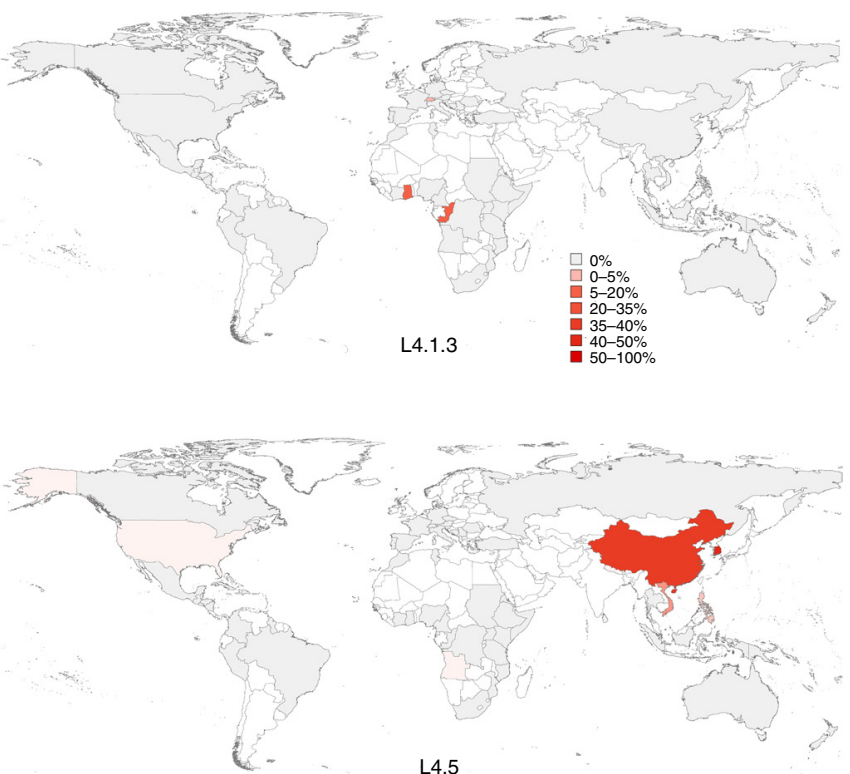

L4.5

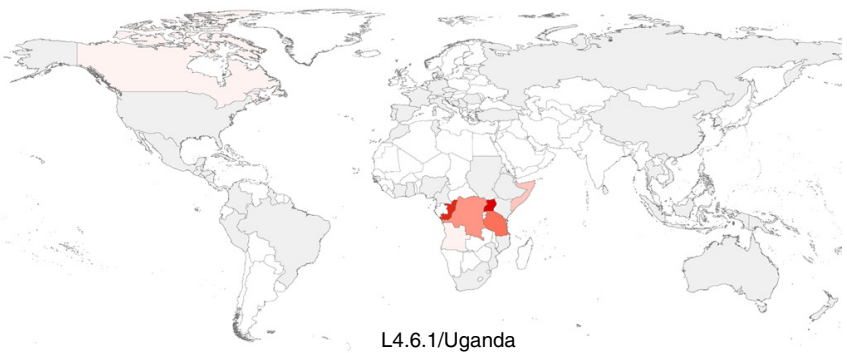

L4.6.1/Uganda

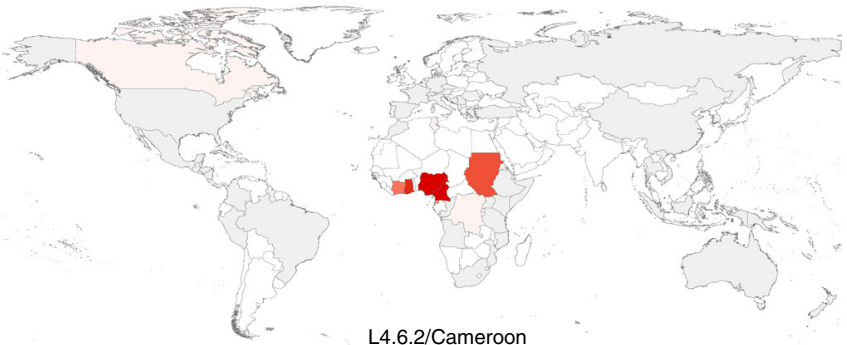

Figure 3 Country-specific proportions of sublineages identify generalists and specialists. (a) The generalist sublineages L4.1.2/Haarlem, L4.3/LAM and L4.10/PGG3 were found globally at high proportions. (b) The locally restricted specialist sublineages L4.1.3/Ghana, L4.5, L4.6.1/Uganda and L4.6.2/Cameroon occurred at high frequencies in only a few countries and were restricted to certain geographical regions. Intensity of red corresponds to the proportion of a sublineage among all lineage 4 isolates in each country. Countries with fewer than three isolates in total are shown as having 'no data' and are filled white. A total of 3,366 lineage 4 isolates were included in this analysis. The color scale for all sublineages is as indicated in a, except for sublineage L4.1.3/Ghana (separate scale shown).

sequences of 293 L4.3/LAM clinical strains representing the global diversity of this sublineage. These were selected from a global collection of 2,132 L4.3/LAM isolates on the basis of standard genotyping data (Supplementary Figs. 7-9 and Supplementary Table 7). For L4.6.1/Uganda, we analyzed whole-genome sequences of 203 clinical strains from Uganda and several neighboring countries (Supplementary Figs. 10 and 11, and Supplementary Table 7). This sample included 28 L4.6.1/Uganda strains identified through screening of 13,067 publically available MTBC whole-genome sequences (Online Methods) ${ }^{44-56}$. Comparing the genetic diversity of these two bacterial populations showed that L4.3/LAM was significantly more diverse than L4.6.1/Uganda (mean of 395 SNPs between pairs of isolates compared to 215 SNPs, respectively; Wilcoxon rank-sum test, $P<0.0001$ ), consistent with the expected difference between generalists and specialists ${ }^{43}$.

\section{Antigenic diversity in lineage 4 sublineages}

We previously reported that, in the human-adapted MTBC, experimentally confirmed human $\mathrm{T}$ cell epitopes were conserved ${ }^{57,58}$. This scenario is unlike that in many other pathogens where genomic regions encoding antigens tend to be diverse as a result of antigenic variation linked to immune escape ${ }^{59}$. When we assessed the evolutionary 

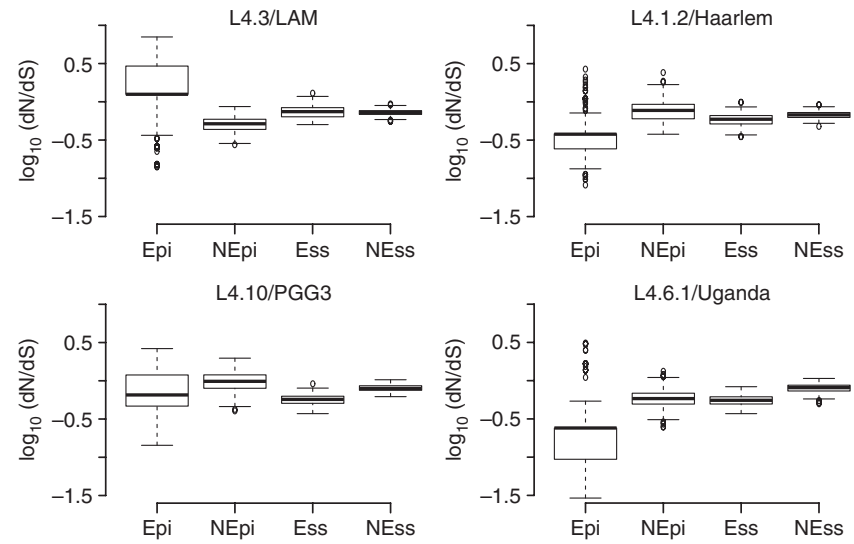

Figure 4 Pairwise ratios of rates of nonsynonymous to synonymous substitutions ( $\mathrm{dN} / \mathrm{dS})$ in generalist and specialist sublineages for different gene categories. Epi, experimentally confirmed human T cell epitopes; nEpi, non-epitope regions of T cell antigens (both obtained from the Immune Epitope Database ${ }^{60}$ ); Ess, essential genes ${ }^{62}$; nEss, nonessential genes ${ }^{62}$. Wilcoxon rank-sum tests: L4.6.1/Uganda $(n=203)$ Epi vs. nEpi, $W=4,952, P<0.001 ;$ L4.6.1/Uganda $(n=203)$ Ess vs. nEss, $W=1,415, P<0.001$; L4.3/LAM ( $n=293$ ) Epi vs. nEpi, $W=74,540, P<0.001 ;$ L4.3/LAM ( $n=293$ ) Ess vs. nEss, $W=45,067$, $P=0.29$; L4.1.2/Haarlem $(n=228)$ Epi vs. nEpi, $W=6,561, P<0.001$; L4.1.2/Haarlem ( $n=228$ ) Ess vs. nEss, $W=13,369, P<0.001$; L4.10/PGG3 ( $n=301$ ) Epi vs. nEpi, $W=27,335, P<0.001$; L4.10/PGG3 ( $n=301)$ Ess vs. nEss, $W=3,103, P<0.001$.

conservation of 1,226 experimentally confirmed human T cell epitopes ${ }^{60}$ in L4.6.1/Uganda by calculating their ratios of nonsynonymous to synonymous substitutions ( $\mathrm{dN} / \mathrm{dS}$ ), we found that these epitopes were significantly more conserved than the non-epitope regions of the corresponding T cell antigens (Wilcoxon rank-sum test, $P<0.0001$; Fig. 4). This result is consistent with our previous findings for the MTBC overall $^{57,58}$. However, for the generalist L4.3/LAM sublineage, we saw the opposite: T cell epitopes showed significantly higher $\mathrm{dN} / \mathrm{dS}$ ratios than non-epitope regions (Wilcoxon rank-sum test, $P<0.0001$; Fig. 4). To test whether a high $\mathrm{dN} / \mathrm{dS}$ ratio in genomic regions encoding $\mathrm{T}$ cell epitopes is characteristic of the generalist sublineages, we analyzed the genomes of 228 L4.2/Haarlem strains and 301 L4.10/PGG3 strains identified by screening of 13,067 publically available genomes (Supplementary Figs. 12 and 13, and Supplementary Table 7). We found that, in contrast to L4.3/LAM, the epitope regions in these generalist sublineages were more conserved than the corresponding non-epitope regions, that is, similar to L4.6.1/Uganda and the MTBC overall $^{57,58}$ (Fig. 4). Consistent with previous reports ${ }^{57,58,61}$, essential genes $^{62}$ were significantly more conserved than nonessential genes in all sublineages, except L4.3/LAM, in which the $\mathrm{dN} / \mathrm{dS}$ ratios of essential and nonessential genes were not significantly different (Fig. 4).

One of the limitations of our $\mathrm{dN} / \mathrm{dS}$ analyses was that, despite a large number of genomes analyzed, within individual sublineages, the mean number of pairwise differences in regions encoding $\mathrm{T}$ cell epitopes was very small (Supplementary Table 8), limiting the accuracy of $\mathrm{dN} / \mathrm{dS}$ inferences for epitopes. Hence, we assessed $\mathrm{T}$ cell epitope diversity by comparing the number of epitopes affected by nonsynonymous variants in the different sublineages (Fig. 5). We found that, in all four sublineages, the majority of epitopes were completely conserved, consistent with our previous findings for the MTBC overall ${ }^{57,58}$. However, each of the three generalist sublineages showed significantly more epitopes harboring at least one amino acid change when compared to the specialist sublineage L4.6.1/Uganda

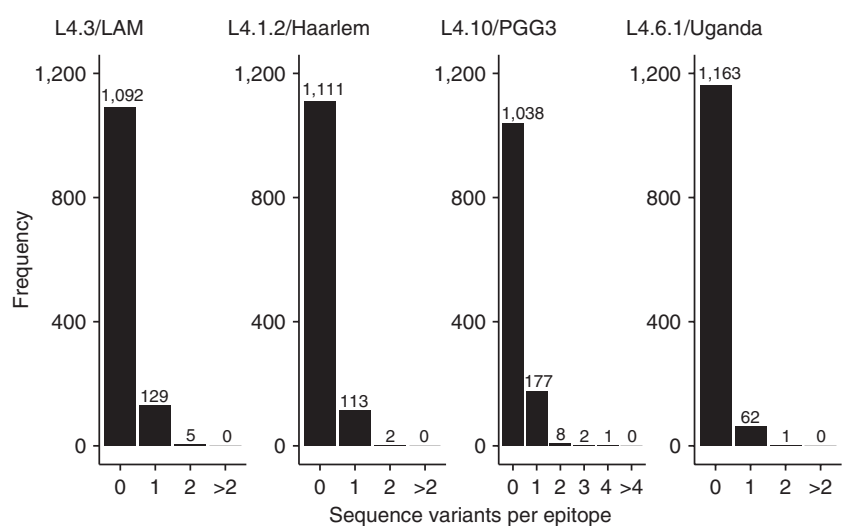

Figure 5 Frequency distribution of the number of epitopes with nonsynonymous variants in generalist and specialist sublineages. A total of 1,226 T cell epitopes were included in the analysis. The number above each bar corresponds to the epitope count. The generalist sublineages are L4.3/LAM, L4.1.2/Haarlem and L4.10/PGG3, while L4.6.1/Uganda is a specialist sublineage. Statistical tests: L4.6.1/Uganda vs. L4.3/LAM, $\chi^{2}=27.04, P<0.001 ;$ L4.6.1/Uganda vs. L4.1.2/Haarlem, $\chi^{2}=15.75$, $P<0.001$; L4.6.1/Uganda vs. L4.1.2/PGG3, $\chi^{2}=68.24, P<0.001$.

( $\chi^{2}$ test, $P<0.0001$ for all comparisons; Fig. 5). It is possible that this comparably higher epitope diversity in generalists might reflect interactions with broader host populations.

The epitopes interrogated in our analysis were encoded by a total of 304 antigens. The number of antigens containing nonsynonymous variation in epitopes was $60,26,46$ and 48 antigens in L4.3/LAM, L4.6.1/Uganda, L4.2/Haarlem and L4.10/PGG3, respectively. When excluding nonsynonymous mutations present only in one strain in each sublineage (which likely represent transient mutations), the number of antigens dropped to 20, 11, 12 and 24 in L4.3/LAM, L4.6.1/ Uganda, L4.2/Haarlem and L4.10/PGG3, respectively (Supplementary Table 9). Interestingly, ten of these antigens exhibited independent, parallel nonsynonymous variation in epitope regions in the different sublineages (Supplementary Table 9). Of these antigens, Pst1, an adhesin promoting phagocytosis ${ }^{63}$, and $\mathrm{FbpB}$, the precursor of the secreted antigen $85-\mathrm{B}^{64}$, had already been identified as encoding diverse epitopes by a previous study in which several MTBC lineages were compared ${ }^{57}$ (Supplementary Table 9). Other antigens exhibiting parallel nonsynonymous changes by different sublineages included known immunodominant secreted antigens such as Mpb64 (ref. 64), MPT32 (ref. 65) and MPT70 (ref. 66) and three latencyassociated antigens (Rv1733c $67, \operatorname{Rv} 3034 c$ and Rv2628 (ref. 68); Supplementary Table 9).

\section{Origin and global spread of the L4.3/LAM sublineage}

Irrespective of the putative biological differences between the lineage 4 sublineages, human migration could also have led to variation in the global distribution of MTBC lineages. Because the most successful sublineage of lineage 4 was also frequently found in Europe, we hypothesized that the global success of L4.3/LAM was driven by European migration and colonization. To test this hypothesis, we first determined the most likely geographical origin of the most recent common ancestor of L4.3/LAM using two methods for reconstruction of ancestral states ${ }^{69}$. By both methods, Europe was predicted as the most likely place of origin for L4.3/LAM (prediction of $100 \%$ and $99.6 \%$ for each method, respectively) (Fig. 6a and Supplementary Fig. 14). Moreover, the ancestral geographical regions reconstructed for subsequent nodes in the phylogeny were consistent with the spread of 
a

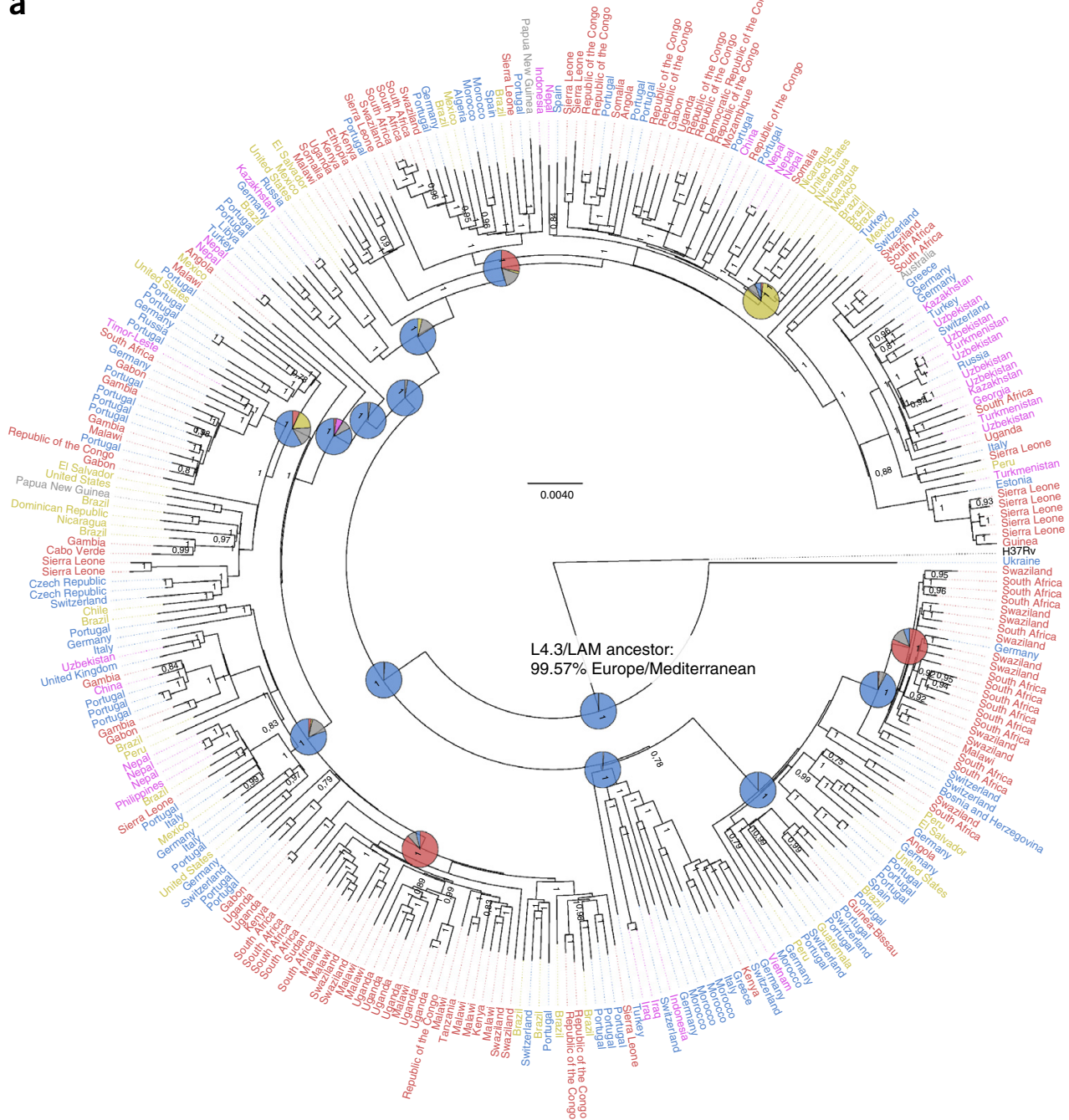

b
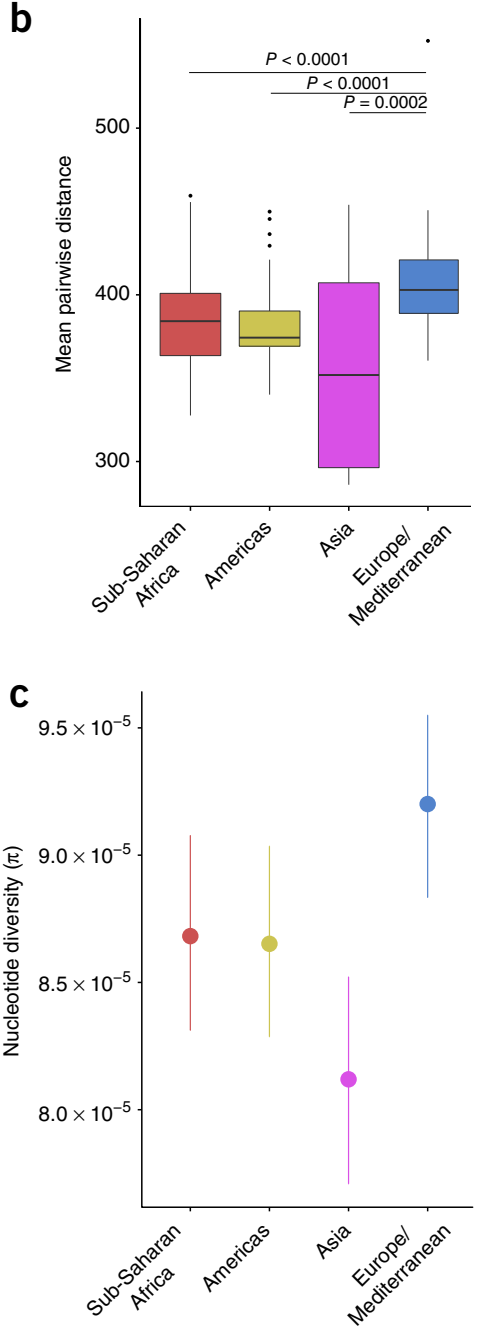

Figure 6 Genome-based phylogeny and diversity by continent of 293 strains of the L4.3/LAM sublineage. (a) Bayesian phylogeny with label colors indicating continent of strain origin: blue, Europe/Mediterranean; red, sub-Saharan Africa; yellow, Americas; pink, Asia. Numbers on nodes indicate posterior probabilities. Pie charts show reconstructed ancestral geographical regions for the internal nodes. The pie colors correspond to the colors of the taxa labels. The hypothetical L4.3/LAM ancestor is labeled; a European origin for this ancestor was supported using a Bayesian method (shown) and a maximum-parsimony method (Supplementary Fig. 14). The scale bar indicates nucleotide substitutions per site. (b) Box plots of pairwise genetic distances (number of polymorphisms) for L4.3/LAM strains by continent ( $P$ values are from Wilcoxon rank-sum tests). Each box corresponds to the $25 \%$ and $75 \%$ quantiles, the black line represents the median and the whiskers extend to 1.5 times the interquartile range. (c) Nucleotide diversity per site $(\pi)$, measured by continent. Error bars indicate 95\% confidence intervals. MTBC isolates from countries of the continent group "Oceania" (UN category; including Australia, New Zealand, Melanesia, Micronesia and Polynesia) were excluded from the genetic diversity analysis in $\mathbf{b}$ and $\mathbf{c}$ because of the low number of samples.

L4.3/LAM from Europe to other parts of the world (Fig. 6a). Finally, we found that L4.3/LAM strains from Europe were genetically more diverse than L4.3/LAM strains from other continents, which further supports a European origin for this sublineage (Kruskall-Wallis test, $P<0.0001$; Fig. 6b,c).

\section{DISCUSSION}

Our findings show that the global success of lineage 4 is a consequence of both biological and social phenomena. Specifically, we found that lineage 4 is genetically diverse and that this diversity is phylogeographically structured. The phylogeography of lineage 4 supports an ecological distinction between globally represented generalists and geographically restricted specialists. Our in-depth population genomic analyses of one specialist and three generalist sublineages showed that, even though the majority of human $\mathrm{T}$ cell epitopes were completely conserved in all four sublineages, the proportion of epitopes with amino acid substitutions was significantly higher in generalists. Finally, we demonstrate a likely European origin for L4.1/LAM, the most frequent and globally widespread generalist sublineage of lineage 4 .

Our observation that lineage 4 is phylogenetically diverse is in line with previous findings $s^{27,70}$ and highlights the importance of large and globally representative samples when studying the population structure of human pathogens. We found that lineage 4 comprises at least ten sublineages, which differ in their geographical distribution. The phylogeography of these sublineages is consistent with an ecological separation into specialists and generalists, with some sublineages showing an intermediate geographical distribution. Our phylogenetic analyses also showed that the three generalist sublineages identified within lineage 4 were not monophyletic (Fig. 1a), indicating that generalism 
was acquired multiple times independently during the evolution of lineage 4 . Specialist sublineages also emerged multiple times, consistent with local adaptation to separate human populations ${ }^{13}$.

One could argue that the reason for specialist sublineages being geographically restricted is that they diverged later than the generalist sublineages during the evolution of lineage 4 and thus had insufficient time to spread globally. However, on the basis of recent findings by Comas et al. ${ }^{71}$, the African specialist sublineages already existed at least several centuries ago, perhaps even several millennia ago, depending on the age of the most recent common ancestor of the MTBC, which has been estimated to be between 70,000 years ${ }^{9,21}$ and 6,000 years ${ }^{72,73}$. Thus, this timespan should have offered ample opportunity for the specialist sublineages to become more geographically widespread.

The genetic diversity of the specialist sublineage L4.6.1/Uganda was significantly lower than that of the generalist sublineage L4.3/LAM, as expected for populations with restricted geographical ranges ${ }^{43}$. Concomitantly, the diversity of T cell epitopes in the specialist sublineage L4.6.1/Uganda was also significantly lower than that in any of the three generalist sublineages analyzed. Whether the low genetic diversity of the specialist sublineage has hindered the adaptation of these strains to other human populations or reflects a restricted niche due to lack of opportunity for spreading will need to be explored in future studies.

In all sublineages analyzed, the large majority of $\mathrm{T}$ cell epitopes were completely conserved, in agreement with previous reports for the MTBC overall ${ }^{57,58}$. This suggests that both generalists and specialists do not use antigenic variation as a main mechanism of immune evasion. Despite this general trend, we found that some antigens have acquired nonsynonymous mutations in parallel in the different sublineages, suggesting that variation in these particular antigens might be beneficial. For example, acquiring nonsynonymous variation may allow particular antigens to be recognized by $\mathrm{T}$ cell receptors of different human populations, which might be beneficial in the presence of different human leukocyte antigen (HLA) alleles ${ }^{58}$. This could also provide an explanation for the differences in the degree of variation in $\mathrm{T}$ cell epitopes between the generalist and specialist sublineages, as generalist sublineages are expected to interact with a broader range of HLA alleles. Alternatively, some nonsynonymous mutations in epitopes might reflect escape from human $\mathrm{T}$ cell recognition ${ }^{58}$. More work is needed to determine whether and how the limited diversity in T cell epitopes in the MTBC is linked to adaptation to different host populations and/or immune escape.

Two independent phylogeographical analyses predicted Europe as the most likely geographical origin for the most recent common ancestor of the L4.3/LAM sublineage. A European origin for L4.3/ LAM was further supported by our finding that strains belonging to this sublineage were more genetically diverse in Europe than in Africa, Asia and the Americas. Taken together, these results suggest a role for Europeans in the spread of L4.3/LAM across the globe. Given the high frequency of L4.3/LAM in Europe (Figs. 2 and 3a)-particularly in patients with tuberculosis from the Iberian Peninsula-and in Latin America ${ }^{74,75}$, Portuguese and Spanish exploration, trade and conquest over the last centuries may have contributed to the global dissemination of this sublineage $\mathrm{e}^{76}$.

Of note, the Americas lack specialist sublineages, including the three African specialist sublineages, despite centuries of slave trade. Notably, this also applies to MTBC lineages 5 and 6 (M. africanum), which today are largely limited to parts of West Africa ${ }^{11}$, the source of most of the African slaves shipped to the Americas. Even if these lineages did reach the Americas at the time, they might have been replaced later by generalist sublineages from Europe, including L4.3/LAM, following the massive influx of Europeans to the Americas during the nineteenth and early twentieth centuries ${ }^{77}$, a time when the European tuberculosis epidemic was at its peak ${ }^{73,78}$. Notably, these human migrations can be viewed as natural experiments in which diverse human populations came into contact with different MTBC genotypes. As mentioned above, there is evidence that the African specialist sublineages of lineage 4 already existed in sub-Saharan Africa centuries ago $^{71}$. Following European contact, generalist sublineages were introduced to Africa, and, today, a significant proportion of human tuberculosis in Africa is caused by L4.1/LAM and other generalist sublineages (Figs. 2 and 3a). By contrast, hardly any spillover of African specialist sublineages has occurred into Europe or populations in the Americas of European ancestry.

Three of the ten sublineages showed an intermediate pattern of geographical distribution. Independent of the open question of whether they might represent generalists or specialists, it is interesting to note that none of these three sublineages were found at high frequency and proportion in Europe. They might therefore represent generalists of non-European origin. Deeper analyses are needed to shed more light on these sublineages.

Our study is limited in that many of the MTBC isolates analyzed come from convenient samples and might therefore not be representative of a particular country. However, for the analysis of sublineage distributions by SNP genotyping, we included more than 3,000 clinical isolates from 100 countries, which should help reduce potential selection bias. For the deep genomic analyses, we selected strains on the basis of a large and diverse collection of classical genotyping patterns and, in addition, screened $>13,000$ MTBC whole-genome sequences available in public repositories. As a further limitation, some isolates in our collection were obtained from patients who recently emigrated from a region of high tuberculosis incidence into a country with low incidence. However, we excluded cases from ongoing transmission and focused on immigrants with reactivation disease: these individuals were most likely infected in their country of origin before moving abroad. Moreover, we used country of birth for all analyses as opposed to country of tuberculosis diagnosis.

In conclusion, our findings indicate that the global success of lineage 4 partly results from the different evolutionary strategies adopted by different sublineages. These strategies reflect an ecological distinction between specialists and generalists. The specialist sublineages are adapted to their sympatric host populations and are geographically restricted. The generalist sublineages exhibit a broader ecological niche and are geographically widespread. Moreover, Europeans contributed to the global spread of the most successful generalist sublineage of lineage 4 . Our results highlight the ecological and epidemiological relevance of the deep phylogenetic diversity within the $\mathrm{MTBC}^{79}$. More generally, exploring potential differences between specialists and generalists in other pathogens will improve understanding of the biology and epidemiology of infectious diseases.

URLs. Immune Epitope Database and Analysis Resource, http://www. iedb.org/; United Nations macro-geographical regions, http://unstats. un.org/unsd/methods/m49/m49regin.htm; DWA-GIS, http://www. diva-gis.org/Data; sciCORE, http://scicore.unibas.ch/; Mesquite, http://mesquiteproject.org/.

\section{METHODS}

Methods, including statements of data availability and any associated accession codes and references, are available in the online version of the paper. 
Note: Any Supplementary Information and Source Data files are available in the online version of the paper.

\section{ACKNOWLEDGMENTS}

We thank S. Lecher, S. Li and J. Zallet for technical support. Calculations were performed at the sciCORE scientific computing core facility at the University of Basel. This work was supported by the Swiss National Science Foundation (grants 310030 166687 (S.G.) and 320030 153442 (M.E.) and Swiss HIV Cohort Study grant 740 to L.F.), the European Research Council (309540-EVODRTB to S.G.), TB-PAN-NET (FP7-223681 to S.N.), PathoNgenTrace projects (FP7-278864-2 to S.N.), SystemsX.ch (S.G.), the German Center for Infection Research (DZIF; S.N.), the Novartis Foundation (S.G.), the Natural Science Foundation of China (91631301 to Q.G.), and the National Institute of Allergy and Infectious Diseases (5U01-AI069924-05) of the US National Institutes of Health (M.E.).

\section{AUTHOR CONTRIBUTIONS}

D.S., D. Brites, L.J., S.N. and S.G. planned the experiments. D.S., L.J., D. Brites, A.T., L.F., L.R., S.B., M. Ballif, Q.L., T.L., Q.G., M.K.-M., M. Bonnet, M.E., R.M., H.M., M.M., G.T.V., J.F., M.G., J.T., F.., J.L.G., A.A.-P., D.Y.-M., E.W., W.S., M.J., W.H.B., I.B., J.B., M.S., S.E.G.V., P. Suffys, A.K., R.W., L.G.-B., B.M., S.D.L., H.-P.B., B.C.d.J., K.T., E.S.-P., M. Bonnet, A.G.-B., M.F., V.N.P.B., K.E., I.A., P.W.N., G.R., F.G., S. Akter, F.N., L.S.-I., N.E.N., A.R., M.H., D.M.C., G.S., S.H., D. Bakonyte, P. Stakenas, R.D., V.C., O.M., S. Al-Hajoj, L.O., F.B., E.J.C., L.D., P. Supply and I.C. contributed reagents and performed the experiments. D.S., L.J., D. Brites, M.C., S.N. and S.G. analyzed the data. D.S., L.J., D. Brites, S.N. and S.G. wrote the manuscript. All authors critically reviewed the manuscript.

\section{COMPETING FINANCIAL INTERESTS}

The authors declare no competing financial interests.

Reprints and permissions information is available online at http://www.nature.com/ reprints/index.html.

1. Futuyma, D.J. \& Moreno, G. The evolution of ecological specialization. Annu. Rev. Ecol. Syst. 19, 207-233 (1988).

2. Woolhouse, M.E., Webster, J.P., Domingo, E., Charlesworth, B. \& Levin, B.R. Biological and biomedical implications of the co-evolution of pathogens and their hosts. Nat. Genet. 32, 569-577 (2002).

3. Woolhouse, M.E., Taylor, L.H. \& Haydon, D.T. Population biology of multihost pathogens. Science 292, 1109-1112 (2001)

4. Kirzinger, M.W. \& Stavrinides, J. Host specificity determinants as a genetic continuum. Trends Microbiol. 20, 88-93 (2012).

5. Vouga, M. \& Greub, G. Emerging bacterial pathogens: the past and beyond. Clin. Microbiol. Infect. 22, 12-21 (2016).

6. Brites, D. \& Gagneux, S. Co-evolution of Mycobacterium tuberculosis and Homo sapiens. Immunol. Rev. 264, 6-24 (2015).

7. Borrell, S. \& Gagneux, S. Infectiousness, reproductive fitness and evolution of drug-resistant Mycobacterium tuberculosis. Int. J. Tuberc. Lung Dis. 13, 1456-1466 (2009).

8. Merker, M. et al. Evolutionary history and global spread of the Mycobacterium tuberculosis Beijing lineage. Nat. Genet. 47, 242-249 (2015).

9. Luo, T. et al. Southern East Asian origin and coexpansion of Mycobacterium tuberculosis Beijing family with Han Chinese. Proc. Natl. Acad. Sci. USA 112, 8136-8141 (2015).

10. Cowley, D. et al. Recent and rapid emergence of W-Beijing strains of Mycobacterium tuberculosis in Cape Town, South Africa. Clin. Infect. Dis. 47, 1252-1259 (2008).

11. de Jong, B.C., Antonio, M. \& Gagneux, S. Mycobacterium africanum-review of an important cause of human tuberculosis in West Africa. PLoS Negl. Trop. Dis. 4, e744 (2010)

12. Firdessa, R. et al. Mycobacterial lineages causing pulmonary and extrapulmonary tuberculosis, Ethiopia. Emerg. Infect. Dis. 19, 460-463 (2013).

13. Gagneux, S. Host-pathogen coevolution in human tuberculosis. Phil. Trans. R. Soc. Lond. B 367, 850-859 (2012).

14. Fenner, L. et al. HIV infection disrupts the sympatric host-pathogen relationship in human tuberculosis. PLoS Genet. 9, e1003318 (2013).

15. Gagneux, S. et al. Variable host-pathogen compatibility in Mycobacterium tuberculosis. Proc. Natl. Acad. Sci. USA 103, 2869-2873 (2006).

16. Baker, L., Brown, T., Maiden, M.C. \& Drobniewski, F. Silent nucleotide polymorphisms and a phylogeny for Mycobacterium tuberculosis. Emerg. Infect. Dis. 10, 1568-1577 (2004).

17. Reed, M.B. et al. Major Mycobacterium tuberculosis lineages associate with patient country of origin. J. Clin. Microbiol. 47, 1119-1128 (2009).

18. Demay, C. et al. SITVITWEB - a publicly available international multimarker database for studying Mycobacterium tuberculosis genetic diversity and molecular epidemiology. Infect. Genet. Evol. 12, 755-766 (2012).

19. Coscolla, M. \& Gagneux, S. Consequences of genomic diversity in Mycobacterium tuberculosis. Semin. Immunol. 26, 431-444 (2014).
20. Coscolla, M. \& Gagneux, S. Does M. tuberculosis genomic diversity explain disease diversity? Drug Discov. Today Dis. Mech. 7, e43-e59 (2010)

21. Comas, I. et al. Out-of-Africa migration and Neolithic coexpansion of Mycobacterium tuberculosis with modern humans. Nat. Genet. 45, 1176-1182 (2013).

22. Coscolla, M. et al. Novel Mycobacterium tuberculosis complex isolate from a wild chimpanzee. Emerg. Infect. Dis. 19, 969-976 (2013).

23. Abadia, E. et al. Resolving lineage assignation on Mycobacterium tuberculosis clinical isolates classified by spoligotyping with a new high-throughput 3R SNPS based method. Infect. Genet. Evol. 10, 1066-1074 (2010).

24. Filliol, I. et al. Global phylogeny of Mycobacterium tuberculosis based on single nucleotide polymorphism (SNP) analysis: insights into tuberculosis evolution, phylogenetic accuracy of other DNA fingerprinting systems, and recommendations for a minimal standard SNP set. J. Bacteriol. 188, 759-772 (2006).

25. Sreevatsan, S. et al. Restricted structural gene polymorphism in the Mycobacterium tuberculosis complex indicates evolutionarily recent global dissemination. Proc. Natl. Acad. Sci. USA 94, 9869-9874 (1997).

26. Homolka, S. et al. High resolution discrimination of clinical Mycobacterium tuberculosis complex strains based on single nucleotide polymorphisms. PLoS One 7, e39855 (2012).

27. Coll, F. et al. A robust SNP barcode for typing Mycobacterium tuberculosis complex strains. Nat. Commun. 5, 4812 (2014).

28. Tsolaki, A.G. et al. Functional and evolutionary genomics of Mycobacterium tuberculosis: insights from genomic deletions in 100 strains. Proc. Natl. Acad. Sci. USA 101, 4865-4870 (2004).

29. Comas, I., Homolka, S., Niemann, S. \& Gagneux, S. Genotyping of genetically monomorphic bacteria: DNA sequencing in Mycobacterium tuberculosis highlights the limitations of current methodologies. PLoS One 4, e7815 (2009).

30. Yeboah-Manu, D. et al. Genotypic diversity and drug susceptibility patterns among M. tuberculosis complex isolates from South-Western Ghana. PLoS One 6, e21906 (2011).

31. Malla, B. et al. First insights into the phylogenetic diversity of Mycobacterium tuberculosis in Nepal. PLoS One 7, e52297 (2012).

32. Fenner, L. et al. Mycobacterium tuberculosis transmission in a country with low tuberculosis incidence: role of immigration and HIV infection. J. Clin. Microbiol. 50, 388-395 (2012).

33. Ballif, M. et al. Genetic diversity of Mycobacterium tuberculosis in Madang, Papua New Guinea. Int. J. Tuberc. Lung Dis. 16, 1100-1107 (2012).

34. Wampande, E.M. et al. Long-term dominance of Mycobacterium tuberculosis Uganda family in peri-urban Kampala-Uganda is not associated with cavitary disease. BMC Infect. Dis. 13, 484 (2013).

35. Ley, S.D. et al. Diversity of Mycobacterium tuberculosis and drug resistance in different provinces of Papua New Guinea. BMC Microbiol. 14, 307 (2014).

36. Stucki, D. et al. Two new rapid SNP-typing methods for classifying Mycobacterium tuberculosis complex into the main phylogenetic lineages. PLoS One 7, e41253 (2012).

37. Bouakaze, C. et al. Matrix-assisted laser desorption ionization-time of flight mass spectrometry-based single nucleotide polymorphism genotyping assay using iPLEX gold technology for identification of Mycobacterium tuberculosis complex species and lineages. J. Clin. Microbiol. 49, 3292-3299 (2011).

8. World Health Organization. Global Tuberculosis Control-Surveillance, Planning, Financing (WHO, 2015).

39. Möller, M., de Wit, E. \& Hoal, E.G. Past, present and future directions in human genetic susceptibility to tuberculosis. FEMS Immunol. Med. Microbiol. 58, 3-26 (2010)

40. Caws, M. et al. The influence of host and bacterial genotype on the development of disseminated disease with Mycobacterium tuberculosis. PLoS Pathog. 4 e1000034 (2008)

41. Asante-Poku, A. et al. Mycobacterium africanum is associated with patient ethnicity in Ghana. PLoS Negl. Trop. Dis. 9, e3370 (2015).

42. Ng, P.C. \& Henikoff, S. SIFT: predicting amino acid changes that affect protein function. Nucleic Acids Res. 31, 3812-3814 (2003).

43. Ellstrand, N.C. \& Elam, D.R. Population genetic consequences of small population size: implications for plant conservation. Annu. Rev. Ecol. Syst. 24, 217-242 (1993)

44. Lanzas, F., Karakousis, P.C., Sacchettini, J.C. \& loerger, T.R. Multidrug-resistant tuberculosis in Panama is driven by clonal expansion of a multidrug-resistant Mycobacterium tuberculosis strain related to the KZN extensively drug-resistan M. tuberculosis strain from South Africa. J. Clin. Microbiol. 51, 3277-3285 (2013).

45. Bryant, J.M. et al Inferring patient to patient transmission of Mycobacterium tuberculosis from whole genome sequencing data. BMC Infect. Dis. 13, 110 (2013).

46. Bryant, J.M. et al. Whole-genome sequencing to establish relapse or re-infection with Mycobacterium tuberculosis: a retrospective observational study. Lancet Respir. Med. 1, 786-792 (2013).

47. Gardy, J.L. et al. Whole-genome sequencing and social-network analysis of a tuberculosis outbreak. N. Engl. J. Med. 364, 730-739 (2011).

48. Casali, N. et al. Evolution and transmission of drug-resistant tuberculosis in Russian population. Nat. Genet. 46, 279-286 (2014).

49. Walker, T.M. et al. Whole-genome sequencing to delineate Mycobacterium tuberculosis outbreaks: a retrospective observational study. Lancet Infect. Dis. 13, 137-146 (2013).

50. Roetzer, A. et al. Whole genome sequencing versus traditional genotyping for investigation of a Mycobacterium tuberculosis outbreak: a longitudinal molecular epidemiological study. PLoS Med. 10, e1001387 (2013). 
51. Farhat, M.R et al. Genomic analysis identifies targets of convergent positive selection in drug-resistant Mycobacterium tuberculosis. Nat. Genet. 45, 1183-1189 (2013).

52. Clark, T.G. et al. Elucidating emergence and transmission of multidrug-resistant tuberculosis in treatment experienced patients by whole genome sequencing. PLoS One 8, e83012 (2013).

53. Jamieson, F.B. et al. Whole-genome sequencing of the Mycobacterium tuberculosis Manila sublineage results in less clustering and better resolution than mycobacterial interspersed repetitive-unit-variable-number tandem-repeat (MIRU-VNTR) typing and spoligotyping. J. Clin. Microbiol. 52, 3795-3798 (2014).

54. Pérez-Lago, L. et al. Whole genome sequencing analysis of intrapatient microevolution in Mycobacterium tuberculosis: potential impact on the inference of tuberculosis transmission. J. Infect. Dis. 209, 98-108 (2014).

55. Guerra-Assunção, J.A. et al. Recurrence due to relapse or reinfection with Mycobacterium tuberculosis: a whole-genome sequencing approach in a large, population-based cohort with a high HIV infection prevalence and active follow-up. J. Infect. Dis. 211, 1154-1163 (2015).

56. Guerra-Assunção, J.A. et al. Large-scale whole genome sequencing of M. tuberculosis provides insights into transmission in a high prevalence area. elife 4, e05166 (2015)

57. Comas, I. et al. Human T cell epitopes of Mycobacterium tuberculosis are evolutionarily hyperconserved. Nat. Genet. 42, 498-503 (2010).

58. Coscolla, M. et al. M. tuberculosis T cell epitope analysis reveals paucity of antigenic variation and identifies rare variable TB antigens. Cell Host Microbe 18, 538-548 (2015)

59. Deitsch, K.W., Lukehart, S.A. \& Stringer, J.R. Common strategies for antigenic variation by bacterial, fungal and protozoan pathogens. Nat. Rev. Microbiol. 7, 493-503 (2009).

60. Ernst, J.D. et al. Meeting Report: NIH Workshop on the Tuberculosis Immune Epitope Database. Tuberculosis (Edinb.) 88, 366-370 (2008).

61. Pepperell, C.S. et al. The role of selection in shaping diversity of natural M. tuberculosis populations. PLoS Pathog. 9, e1003543 (2013).

62. Zhang, Y.J. et al. Global assessment of genomic regions required for growth in Mycobacterium tuberculosis. PLoS Pathog. 8, e1002946 (2012).

63. Esparza, M. et al. PstS-1, the 38-kDa Mycobacterium tuberculosis glycoprotein, is an adhesin, which binds the macrophage mannose receptor and promotes phagocytosis. Scand. J. Immunol. 81, 46-55 (2015).

64. Bekmurzayeva, A., Sypabekova, M. \& Kanayeva, D. Tuberculosis diagnosis using immunodominant, secreted antigens of Mycobacterium tuberculosis. Tuberculosis (Edinb.) 93, 381-388 (2013).
65. Nagai, S., Wiker, H.G. Harboe, M. \& Kinomoto, M. Isolation and partial characterization of major protein antigens in the culture fluid of Mycobacterium tuberculosis. Infect. Immun. 59, 372-382 (1991).

66. Juárez, M.D., Torres, A. \& Espitia, C. Characterization of the Mycobacterium tuberculosis region containing the mpt83 and mpt70 genes. FEMS Microbiol. Lett. 203, 95-102 (2001).

67. Coppola, M. et al. Synthetic long peptide derived from Mycobacterium tuberculosis latency antigen Rv1733c protects against tuberculosis. Clin. Vaccine Immunol. 22 1060-1069 (2015).

68. Araujo, LS. et al. Profile of interferon- $\gamma$ response to latency-associated and nove in vivo expressed antigens in a cohort of subjects recently exposed to Mycobacterium tuberculosis. Tuberculosis (Edinb.) 95, 751-757 (2015).

69. Yu, Y., Harris, A.J., Blair, C. \& He, X. RASP (Reconstruct Ancestral State in Phylogenies): a tool for historical biogeography. Mol. Phylogenet. Evol. 87, 46-49 (2015).

70. Brudey, K. et al. Mycobacterium tuberculosis complex genetic diversity: mining the fourth international spoligotyping database (SpolDB4) for classification, population genetics and epidemiology. BMC Microbiol. 6, 23 (2006).

71. Comas, I. et al. Population genomics of Mycobacterium tuberculosis in Ethiopia contradicts the virgin soil hypothesis for human tuberculosis in sub-Saharan Africa. Curr. Biol. 25, 3260-3266 (2015).

72. Bos, K.I. et al. Pre-Columbian mycobacterial genomes reveal seals as a source of New World human tuberculosis. Nature 514, 494-497 (2014).

73. Kay, G.L. et al. Eighteenth-century genomes show that mixed infections were common at time of peak tuberculosis in Europe. Nat. Commun. 6, 6717 (2015).

74. Lazzarini, L.C. et al. Discovery of a novel Mycobacterium tuberculosis lineage that is a major cause of tuberculosis in Rio de Janeiro, Brazil. J. Clin. Microbiol. 45, 3891-3902 (2007).

75. Perdigão, J. et al. Unraveling Mycobacterium tuberculosis genomic diversity and evolution in Lisbon, Portugal, a highly drug resistant setting. BMC Genomics 15 , 991 (2014)

76. Mokrousov, I. et al. Latin-American-Mediterranean lineage of Mycobacterium tuberculosis: human traces across pathogen's phylogeography. Mol. Phylogenet. Evol. 99, 133-143 (2016).

77. Baily, S.L. \& Miguez, E.J. Mass Migration to Modern Latin America. (Scholarly Resources, 1993).

78. Bates, J.H. \& Stead, W.W. The history of tuberculosis as a global epidemic. Med. Clin. North Am. 77, 1205-1217 (1993).

79. Gagneux, S. \& Small, P.M. Global phylogeography of Mycobacterium tuberculosis and implications for tuberculosis product development. Lancet Infect. Dis. 7, 328-337 (2007)

${ }^{1}$ Swiss Tropical and Public Health Institute, Basel, Switzerland. ${ }^{2}$ University of Basel, Basel, Switzerland. ${ }^{3}$ Forschungszentrum Borstel, Borstel, Germany. ${ }^{4}$ Institut Pasteur de Tunis, Université de Tunis El Manar, Tunis, Tunisia. ${ }^{5}$ Key Laboratory of Medical Molecular Virology of the Ministries of Education and Health, Institutes of Biomedical Sciences and Institute of Medical Microbiology, School of Basic Medical Sciences, Fudan University, Shanghai, China. ${ }^{6}$ Institute for Socia and Preventive Medicine, University of Bern, Bern, Switzerland. ${ }^{7}$ Laboratory of Infection and Immunity, School of Basic Medical Science, West China Center of Medical Sciences, Sichuan University, Chengdu, China. ${ }^{8}$ School of Medicine, University of California at San Francisco, San Francisco, California, USA. ${ }^{2}$ aboratório de Saúde Pública, Lisbon, Portugal. ${ }^{10}$ Hospital Nossa Senhora da Paz, Cubal, Angola. ${ }^{11}$ Servei de Microbiologia, Hospital Clínic-ISGlobal, Barcelona, Spain.

12 Victorian Infectious Diseases Reference Laboratory, Melbourne, Victoria, Australia. ${ }^{13}$ Ifakara Health Institute, Bagamoyo, Tanzania. ${ }^{14}$ Public Health Ontario, Toronto, Ontario, Canada. ${ }^{15}$ Noguchi Memorial Institute for Medical Research, University of Ghana, Accra, Ghana. ${ }^{16}$ Department of Medical Microbiology, Makerere University, Kampala, Uganda. ${ }^{17}$ Department of Global Health, University of Amsterdam, Amsterdam, the Netherlands. ${ }^{18}$ Department of Molecular Biology and Microbiology, Case Western Reserve University, Cleveland, Ohio, USA. ${ }^{19}$ LabPlus, Auckland City Hospital, Auckland, New Zealand. ${ }^{20}$ Life and Health Sciences Research Institute (ICVS), School of Health Sciences, University of Minho, Braga, Portugal. ${ }^{21}$ ICVS/3B's-PT Government Associate Laboratory, Braga/Guimarães, Portugal. 22Oswaldo Cruz Institute, Rio de Janeiro, Brazil. ${ }^{23}$ Institute of Infectious Disease and Molecular Medicine, and Department of Clinical Laboratory Sciences, University of Cape Town, Cape Town, South Africa. ${ }^{24}$ Department of Medicine, Imperial College London, London, UK. ${ }^{25}$ Francis Crick Institute Mill Hill Laboratory, London, UK. ${ }^{26}$ Papua New Guinea Institute of Medical Research, Goroka, Papua New Guinea. ${ }^{27}$ Insitute of Tropical Medicine, Antwerp, Belgium. ${ }^{28}$ Tartu University Hospital United Laboratories, Mycobacteriology, Tartu, Estonia. ${ }^{29}$ Clinical Research Department, Epicentre, Paris, France. ${ }^{30}$ Department of Microbiology, University Hospital La Fe, Valencia, Spain. 31 Institute of Tropical Medicine, University of Tübingen, Tübingen, Germany. ${ }^{32}$ Institute Laboratory for Tuberculosis Research (LTR), Biotechnology Center (BTC), University of Yaoundé I, Yaoundé, Cameroon. ${ }^{33}$ Department of Pathology, University of Arkansas for Medical Sciences, Little Rock, Arkansas, USA. ${ }^{34}$ Department of Medical Laboratory Technology, Faculty of Medical Technology, Baghdad, Iraq. ${ }^{35}$ Institute of Tropical Medicine and Infectious Diseases (ITROMID), Jomo Kenyatta University of Agriculture and Technology (JKUAT), Nairobi, Kenya. ${ }^{36}$ Department of Pathology, Aga Khan University Hospital (AKUH), Nairobi, Kenya. ${ }^{37}$ Medical Research Council, Fajara, the Gambia. ${ }^{38}$ Fondation Congolaise pour la Recherche Médicale, Université Marien Gouabi, Brazzaville, Republic of the Congo. ${ }^{39}$ Right to Care and the Clinical HIV Research Unit, University of the Witwatersrand, Johannesburg, South Africa. ${ }^{40}$ National Institute of Medical Research, Mbeya Medical Research Centre (NIMR-MMRC), Mbeya, Tanzania. ${ }^{41}$ Division of Infectious Diseases and Tropical Medicine, Medical Centre of the University of Munich, and German Centre for Infection Research (DZIF), partner site Munich, Munich, Germany. ${ }^{42}$ Emerging Bacterial Pathogens Unit, IRCCS, San Raffaele Scientific Institute, Milan, Italy. ${ }^{43}$ Riga East University Hospital, Centre of Tuberculosis and Lung Diseases, Riga, Latvia. ${ }^{44}$ WHO Supranational TB Reference Laboratory, Department of Microbiology, Public Health Agency of Sweden, Solna, Sweden. ${ }^{45}$ Department of Immunology and Cell Biology, Institute of Biotechnology, Vilnius University, Vilnius, Lithuania. ${ }^{46}$ Institute for Epidemiology, Schleswig-Holstein University Hospital, Kiel, Germany. ${ }^{47}$ National Tuberculosis Reference Laboratory, Phthysiopneumology Institute, Chisinau, Moldova. ${ }^{48}$ 'Marius Nasta' Pneumophtisiology Institute, Bucharest, Romania. ${ }^{49}$ Department of Infection and Immunity, King Faisal Specialist Hospital and Research Centre, Riyadh, Saudi Arabia. ${ }^{50}$ Instituto de Medicina Tropical Alexander von Humboldt, Molecular Epidemiology Unit-Tuberculosis, Universidad Peruana Cayetano Heredia, Lima, Peru. ${ }^{51}$ Warren Alpert School of Medicine at Brown University, Miriam Hospital, Providence, Rhode Island, USA. 52 Moi University School of Medicine, Eldoret, Kenya. ${ }^{53}$ Université Lille, CNRS, INSERM, CHU Lille, Institut Pasteur de Lille, U1019, UMR 8204, CIIL, Centre d'Infection et d'Immunité de Lille, Lille, France. ${ }^{54}$ Institute of Biomedicine of Valencia (IBV-CSIC), Valencia, Spain. ${ }^{55} \mathrm{CIBER}$ Epidemiology and Public Health, Madrid, Spain. ${ }^{56}$ German Center for Infection Research, Borstel Site, Borstel, Germany. ${ }^{57}$ These authors contributed equally to this work. Correspondence should be addressed to S.G. (sebastien.gagneux@unibas.ch). 


\section{ONLINE METHODS}

Mycobacterial isolates. For the definition of lineage 4 sublineages, we used 72 whole-genome sequences of MTBC lineage 4 and reference sequences for the other MTBC lineages published previously ${ }^{21,22}$ (Supplementary Table 7). These represented the largest collection of lineage 4 whole-genome sequences available at that time. For SNP screening of clinical isolates for sublineage classification, we used a retrospective global collection of 3,366 MTBC lineage 4 isolates from 100 countries (Supplementary Table 5) ${ }^{15,30-35}$. All isolates had previously been identified as MTBC lineage 4 by SNP typing, genomic deletion analysis or spoligotyping. Approximately one-third of these isolates were from patients who migrated to another country $(1,106 ; 32.9 \%)$, and we used country of birth for each patient as a proxy for the origin of the MTBC strains. Two-thirds of the isolates $(2,260 ; 67.1 \%)$ were from patients for whom the country of isolation and country of birth were the same. Isolates of L4.6.1/Uganda from Uganda were genotyped in our previous work ${ }^{34}$. For the in-depth population genomic analysis of L4.3/LAM, we included previously published genomes ${ }^{21,27,44}$ and generated whole-genome sequences of additional strains selected from a large collection of 2,132 MIRU-VNTR-genotyped isolates representing the global diversity of L4.3/ LAM (Supplementary Fig. 7). Starting from 500 whole-genome sequences, we excluded sequences with bad quality (sequencing coverage $<15 \times$ or proportion of homozygous variant calls $<85 \%$ ), isolates in transmission clusters (defined as isolate pairs differing by $\leq 12 \mathrm{SNPs}$ ) and strains with unknown country of origin, resulting in whole-genome sequencing data for 293 L4.3/LAM strains, which were included in the final analysis (Supplementary Table 7). For the in-depth population genomic analyses of L4.6.1/Uganda, we generated whole-genome sequencing data for 175 isolates of the L4.6.1/Uganda genotype, selected for maximal geographical diversity and from previous studies ${ }^{34}$. Moreover, to further increase geographical coverage and genetic diversity among L4.6.1/Uganda strains, we analyzed all available whole-genome sequencing data from several published studies ${ }^{8,45-56,75}$ and other whole-genome data available in the public domain. We used KvarQ ${ }^{80}$ to screen for the L4.6.1/Uganda-specific SNPs described below. Starting from 13,067 genome sequences and excluding all clustered isolates except for one representative of each cluster, we identified 28 additional L4.6.1/Uganda genome sequences that we included in our analysis of a total of 203 genomes (Supplementary Table 7). For genomic analysis of L4.1.2/Haarlem and L4.10/PGG3 strains, we screened the same 13,067 isolates (plus our own collection) for clade-specific SNPs for these two sublineages. We identified 505 genome sequences for L4.1.2/Haarlem and 748 sequences for L4.10/PGG3. After excluding problematic sequences and strains in transmission clusters (for criteria, see above), we used 228 strains from L4.1.2/Haarlem and 301 strains from L4.10/PGG3. H37Rv was used as the outgroup for all sublineage phylogenies except that for L4.10/PGG3, for which an isolate from L4.1.2/Haarlem was used (H37Rv belongs to L4.10/PGG3).

Whole-genome sequencing, variant calling and filtering. Whole-genome sequencing of new MTBC isolates was performed using Illumina chemistry (MiSeq, HiSeq 2000/2500, NextSeq; paired end or single end). Illumina MiSeqgenerated sequencing reads were clipped for adaptors with Trimmomatic ${ }^{81}$ before mapping. We used a previously described pipeline for the mapping of short sequencing reads to the reference genome (a reconstructed hypothetical MTBC ancestor) with BWA 0.6.2 (ref. 21). SNPs were called with SAMtools 0.1 .19 and excluded if the coverage was less than $10 \%$ or more than $200 \%$ of the average coverage for the genome, if not supported by at least two reads on each strand or if the quality was less than 30. All SNPs were then annotated using H37Rv reference annotation (AL123456.2) with ANNOVAR ${ }^{82}$ and customized scripts. SNPs in regions annotated as 'PE/PPE/PGRS', 'maturase', 'phage' or 'insertion sequence' were excluded. Additionally, we excluded SNPs in genes with previously identified repetitive regions ${ }^{58}$. Small insertions and deletions called by BWA/SAMtools as 'INDEL' were not considered for the analyses. The presence of large genomic deletions reported previously ${ }^{15,28,74}$ was assessed by manually inspecting BAM alignment files from BWA mappings in Artemis for the presence of reads in the genomic regions with described deletions. Alternatively, we used a new test suite in $\mathrm{KvarQ}^{80}$ to check for reads aligning to 25-bp query sequences of the corresponding deletion.

Phylogenetic and population genetic analyses for the definition of sublineages. A phylogenetic tree was generated with all lineage 4 genomes, plus several reference genomes from all other MTBC lineages. Pairwise SNP distances were calculated using MEGA5 (ref. 83) and the ape package in $\mathrm{R}$ (ref. 84). $F_{\mathrm{ST}}$ values (estimation of population separation) were calculated using Arlequin 3.5 (ref. 85). Statistical significance was obtained by permutating haplotypes between sublineages. Principal-component analysis was carried out with Jalview ${ }^{86}$. Naming of the lineage 4 sublineages was adapted to that of Coll et al. ${ }^{27}$ whenever possible. However, in that publication, no criteria for the definition of sublineages were given and not all sublineages were identified as such. We therefore added continuous numbers for the clades that were not defined by Coll et al. The new sublineages defined in this study are L4.1.3/Ghana and L4.10/PGG3 (with the latter including L4.5, L4.8 and L4.9 according to the nomenclature from Coll et al.). The full phylogenetic tree, including previous markers and spoligotyping family names, is shown in Supplementary Figure 1.

Identification of sublineage-specific SNPs. The alignment of all SNPs from the initial 72 MTBC lineage 4 strains was imported into Mesquite, in parallel with the phylogenetic tree generated from the same data in MEGA5. We used the 'Trace Character History' module of Mesquite to map polymorphisms to clades. The full data set of reconstructed positions was exported, and sublineage-specific SNPs were extracted as nucleotide differences between internal nodes of the phylogeny.

SNP typing to screen for MTBC lineage 4 sublineages. We developed a new SNP typing assay to screen clinical isolates for the defined lineage 4 sublineages. For this, we selected one 'diagnostic' SNP per sublineage using previously defined methods and criteria ${ }^{36}$. Oligonucleotides were designed for a 10-plex MOL-PCR assay based on the Luminex xTag platform (Luminex) (Supplementary Table 2) ${ }^{36}$. DNA extracts from clinical MTBC isolates were then screened with (i) the new MOL-PCR assay, (ii) standard PCR amplification and subsequent Sanger sequencing of the region up- and downstream of the sublineage-specific SNP (see Supplementary Table 4 for PCR and sequencing primers), (iii) a real-time PCR melting curve assay using the same SNPs (Supplementary Table 2), or (iv) the MassARRAY platform (Sequenom) using phylogenetically redundant SNPs (Supplementary Table 3). The set of SNPs used in the MassARRAY typing scheme covered only six of the ten sublineages. Hence, all lineage 4 isolates for which none of the six SNPs in the MassARRAY typing had the mutant allele $(n=49)$ were subjected to the Luminex assay described above. For all isolates, patient place of birth was used as country information. We obtained sublineage classification data for 3,273 (97.2\%) of a total of 3,366 isolates (Supplementary Table 5)

Spatial analysis and data presentation. For each country with lineage 4 sublineage data available, sublineage proportions (in comparison to all lineage 4 isolates from the same country) were calculated and mapped to a world map with ArcGIS ArcMap 10.0 (Esri). A shapefile with country boundaries was used from DIVA-GIS, which is freely available. Categories for number of countries were defined as $0,1-3,4-10$ and $>10$ countries. For individual sublineage 'heat maps', countries with fewer than three isolates were not included. For the additional maps shown in Supplementary Figure 6, sublineage proportions were normalized by the tuberculosis prevalence in the country, as estimated by the World Health Organization (WHO ${ }^{38}$, and the area of the country. Other figures were generated with the ggplot2 library in R and with GraphPad Prism 6.02 (GraphPad Software). Statistical analyses were performed with R or GraphPad Prism.

SIFT analyses of the functional effects of fixed sublineage-specific SNPs. SNPs fixed in each of the ten sublineages were assessed for predicted functional consequence with SIFT in the software SIFT4G (v2.1 $)^{42}$ and the precompiled M. tuberculosis database GCA_000195955.2.22. Conservation levels of SNPs in the precompiled database had been obtained by comparing $M$. tuberculosis $\mathrm{H} 37 \mathrm{Rv}$ proteins to all proteins in the UniRef ${ }^{87}$ database. We pooled SNPs fixed in the generalist sublineages and the specialist sublineages separately and excluded the L4.1.2/Ghana sublineage, as whole-genome sequences for only two very closely related isolates were available. Gene categories were analyzed on the basis of the classification by Tuberculist ${ }^{88}$. 
Phylogenetic reconstruction and population genetic analyses. The final alignment of polymorphic positions in all strains was used to estimate phylogenies with Bayesian methods using MrBayes 3.2.5 (ref. 89) for the L4.3/ LAM and L4.6.1/Uganda sublineages (Fig. 6 and Supplementary Fig. 10). For the Bayesian analysis, we used a gamma rate distribution estimated from our data set and a burn-in equal to one-tenth the number of generations; after the burn-in phase, every 100th tree was saved. Two parallel Markov chains were run in each of two runs. The tree length, log-likelihood score and alpha value of the gamma distribution were inspected for stationarity before termination of MrBayes. Trees were generated with standard parameters. A consensus tree was used for further analyses. Additionally, we used MEGA5 (ref. 83) to generate maximum-likelihood phylogenetic trees (Supplementary Figs. 9 and 11-13). We used the general time-reversible (GTR) model of evolution and 500 pseudoreplicates for bootstrapping confidence levels. Positions with gaps in more than $50 \%$ of the taxa were ignored. Tree figures were generated using FigTree version 1.4.2. Pairwise SNP distances were calculated with the ape package and the dna.dist function in $\mathrm{R}$ version 3.2.2, using raw counts of mutations and pairwise deletions for sites with gaps. For the comparison of pairwise number-of-SNP distributions overall (L4.3/LAM and L4.6.1/Uganda) and between continents for L4.3/LAM, a mean SNP distance to all isolates of the same population was calculated for each isolate and a distribution of the mean pairwise distance was plotted. Wilcoxon rank-sum and Kruskall-Wallis tests were used to test for differences between continents, as data were assumed not to be normally distributed. Average pairwise nucleotide diversities per site $(\pi)$ were calculated as the average number of pairwise mismatches among a set of sequences divided by the total length of the interrogated sequences in base pairs (equation (4).21 in ref. 87). Confidence intervals for $\pi$ were obtained through bootstrapping (1,000 replicates) by resampling with replacement the nucleotide sites of the original alignments of polymorphic positions using the function sample in R. Lower and upper levels of confidence were obtained by calculating the 2.5th and 97.5th quantiles of the $\pi$ distribution obtained by bootstrapping. Code details are available upon request.

Antigenic diversity in human T cell epitopes. Experimentally confirmed human MTBC T cell epitope sequences were retrieved from the Immune Epitope Database on 24 April 2015. Only linear epitopes from the MTBC (77643) tested in human T cell assays, with no major histocompatibility complex (MHC) restrictions, were selected (1,730 epitopes). The sequence of each epitope was searched by BLASTP ${ }^{90}$ against the reference strain $(\mathrm{H} 37 \mathrm{Rv})$ to obtain genomic coordinates. Epitopes with no coordinates in $\mathrm{H} 37 \mathrm{Rv}$ or for which no accurate coordinates could be determined (owing to multiple hits) and epitopes in repetitive regions such as PE/PPE genes, phage-related genes and transposases were excluded, rendering a final set of 1,226 epitopes. These epitopes are distributed across 304 antigens and have some overlapping sequences. To proceed with the sequence analysis, alignments were obtained by concatenating all epitope sequences after excluding sequence redundancy. Alignments of non-epitope-containing antigens were obtained by excluding the regions described as epitopes for each respective antigen. To assess how other regions of the genome are evolving, alignments for essential and nonessential genes were also obtained ${ }^{62}$.

Alignments of epitopes and non-epitope-containing regions for antigens, as well as essential and nonessential genes, were used to calculate pairwise $\mathrm{dN} / \mathrm{dS}$ ratios for the L4.3/LAM, L4.6.1/Uganda, L4.10/PGG3 and L4.1.2/Haarlem sublineages. The $\mathrm{dN} / \mathrm{dS}$ measures were calculated using all polymorphic sites within each sublineage and therefore reflect both within-sublineage substitutions and transient polymorphisms. Pairwise $\mathrm{dN}$ and $\mathrm{dS}$ values within each sublineage were calculated using the R package seqinr with the kaks function.
To avoid having undetermined pairwise $\mathrm{dN} / \mathrm{dS}$ values due to $\mathrm{dN}$ or $\mathrm{dS}$ being zero, a mean $\mathrm{dN} / \mathrm{dS}$ value was then calculated for each sequenced isolate by dividing its mean pairwise $\mathrm{dN}$ by its mean pairwise $\mathrm{dS}$ with respect to all other sequenced isolates within each sublineage. The statistical differences between the epitopes and non-epitope regions of antigens within each sublineage were accessed using Wilcoxon rank-sum tests with continuity correction implemented in $\mathrm{R}$ version 3.2.2.

Reconstruction of the geographical origin of L4.3/LAM. The software RASP $^{69}$ was used to reconstruct the hypothetical geographical origin of the MTBC L4.3/LAM ancestor genotype. The Bayesian phylogeny of 294 isolates (including H37Rv as the outgroup) and the corresponding continents of birth for patients were loaded as a distribution. We used the S-DIVA (a parsimonybased method) as well as the Bayesian binary method (BBM) implementation in RASP. A set of trees from MrBayes ${ }^{89}$ was used to correct for phylogenetic uncertainty in the S-DIVA analysis. Populations were defined according to country of birth of the patients and according to United Nations definition. The isolates from Turkey, Libya, Algeria and Morocco were in the category 'Europe and Mediterranean'. RASP reconstruction was performed without the outgroup (H37Rv). As we observed a single strain (from Ukraine) with a distinct, basal position in the phylogeny, we also performed a sensitivity analysis by excluding that isolate for the RASP analysis. With both methods, $\mathrm{BBM}$ as well as S-DIVA, the changes in the proportion of strains from each continent were minor. With BBM, the proportion of 'Europe/Mediterranean' for the 'L4.3/LAM ancestor' decreased to $98.8 \%$, and with S-DIVA the proportion of 'Europe/Mediterranean' decreased to $99.0 \%$ when excluding this basal isolate.

Data availability statement. All data generated or analyzed during this study are included in this published article and its supplementary information files. Sequencing reads have been submitted to the EMBL-EBI European Nucleotide Archive (ENA) Sequence Read Archive (SRA) under study accession PRJEB11460. Sample and run accession numbers for individual strains can be found in Supplementary Table 7.

80. Steiner, A., Stucki, D., Coscolla, M., Borrell, S. \& Gagneux, S. KvarQ: targeted and direct variant calling from fastq reads of bacterial genomes. BMC Genomics 15, 881 (2014).

81. Bolger, A.M., Lohse, M. \& Usadel, B. Trimmomatic: a flexible trimmer for Illumina sequence data. Bioinformatics 30, 2114-2120 (2014).

82. Wang, K., Li, M. \& Hakonarson, H. ANNOVAR: functional annotation of genetic variants from high-throughput sequencing data. Nucleic Acids Res. 38, e164 (2010).

83. Tamura, K. et al. MEGA5: molecular evolutionary genetics analysis using maximum likelihood, evolutionary distance, and maximum parsimony methods. Mol. Biol. Evol. 28, 2731-2739 (2011)

84. Paradis, E., Claude, J. \& Strimmer, K. APE: Analyses of Phylogenetics and Evolution in R language. Bioinformatics 20, 289-290 (2004).

85. Excoffier, L., Laval, G. \& Schneider, S. Arlequin (version 3.0): an integrated software package for population genetics data analysis. Evol. Bioinform. Online 1, 47-50 (2007).

86. Waterhouse, A.M., Procter, J.B., Martin, D.M., Clamp, M. \& Barton, G.J. Jalview Version 2-a multiple sequence alignment editor and analysis workbench. Bioinformatics 25, 1189-1191 (2009).

87. Hartl, D. \& Clarck, A.G. Principles of Population Genetics (Sinauer, 2007).

88. Lew, J.M., Kapopoulou, A., Jones, L.M. \& Cole, S.T. TubercuList-10 years after. Tuberculosis (Edinb.) 91, 1-7 (2011).

89. Ronquist, F. \& Huelsenbeck, J.P. MrBayes 3: Bayesian phylogenetic inference under mixed models. Bioinformatics 19, 1572-1574 (2003).

90. Altschul, S.F., Gish, W., Miller, W., Myers, E.W. \& Lipman, D.J. Basic local alignment search tool. J. Mol. Biol. 215, 403-410 (1990). 